\title{
On Preemptive Scheduling on Uniform Machines to Minimize Mean Flow Time
}

\author{
Svetlana A. Kravchenko ${ }^{1}$ \\ United Institute of Informatics Problems, \\ Surganova St. 6, 220012 Minsk, Belarus \\ kravch@newman. bas-net. by \\ Frank Werner \\ Otto-von-Guericke-Universität, Fakultät für Mathematik, \\ 39106 Magdeburg, Germany \\ Frank. Werner@Mathematik. Uni-Magdeburg. De
}

July 28,2008

\begin{abstract}
In this paper we give a polynomial algorithm for problem $Q \mid r_{j}, p_{j}=$ $p$, pmtn $\mid \sum C_{j}$ whose complexity status was open yet. The algorithm is based on a reduction of the scheduling problem to a linear program. The crucial condition for implementing the proposed reduction is the known order of job completion times.
\end{abstract}

Keywords: parallel uniform machines, linear programming, maximum flow, polynomial algorithm

\section{Introduction}

The problem considered can be stated as follows. There are $n$ independent jobs and $m$ parallel uniform machines. For each job $J_{j}, j=1, \ldots, n$, we know its processing time

\footnotetext{
${ }^{1}$ Supported by the Alexander von Humboldt Foundation
} 
$p_{j}=p$ and its release time $r_{j} \geq 0$. Each machine $M_{q}, q=1, \ldots, m$, has some speed $s_{q}$, i.e. the execution of job $J_{j}$ on machine $M_{q}$ requires $p / s_{q}$ time units. Any machine can process only one job at a time, and at any moment any job can be processed only by one but arbitrary machine. Preemptions of processing are allowed, i.e. the processing of any job may be interrupted at any time and resumed later, possibly on a different machine. We assume that all numerical data $r_{j}, p, s_{q}$ are integers. For a schedule $s$, let $C_{j}(s)$ denote the time at which the processing of job $J_{j}$ is completed. If no ambiguity arises, we drop the reference to schedule $s$ and write $C_{j}$. The problem is to schedule all jobs so as to minimize the optimality criterion $\sum_{j=1}^{n} C_{j}$. The described problem can be denoted as $Q\left|r_{j}, p_{j}=p, \operatorname{pmtn}\right| \sum C_{j}$.

Note that problem $P \mid r_{j}$, pmtn $\mid \sum C_{j}$ and, therefore, also problem $Q \mid r_{j}$, pmtn $\mid$ $\sum C_{j}$ are unary NP-hard [1] whereas problem $Q \mid r_{j}$, pmtn $\mid C_{\max }$ can be solved in $O(n \log n+m n)$ time [3]. Problem $R\left|r_{j}, \operatorname{pmtn}\right| L_{\max }$ can be effectively solved by reducing it to linear programming [5]. Problem $R|\operatorname{pmtn}| \sum C_{j}$ is NP-hard in the strong sense, and problem $R\left|p_{j i} \in\left\{p_{j}, \infty\right\}\right| \sum C_{j}$ can be solved in $O\left(n^{3}\right)$ time [6].

The main result in this paper is a polynomial algorithm for problem $Q \mid r_{j}, p_{j}=p$, pmtn $\mid$ $\sum C_{j}$. This result is a generalization of the polynomial algorithm for problem $P \mid r_{j}, p_{j}=$ $p$, pmtn $\mid \sum C_{j}$ from paper [2]. Throughout the paper, we suppose that the jobs are enumerated in such a way that $r_{1} \leq \ldots \leq r_{n}$ holds. Furthermore, we assume that $r_{1}=0$.

\section{A polynomial algorithm for problem $Q \mid r_{j}, p_{j}=$ $p, \operatorname{pmtn} \mid \sum C_{j}$}

In this section, we derive a polynomial algorithm for problem $Q\left|r_{j}, p_{j}=p, \operatorname{pmtn}\right| \sum C_{j}$.

Statement 1 For problem $Q \mid r_{j}, p_{j}=p$, pmtn $\mid \sum C_{j}$ an optimal schedule can be found in the class of schedules, for which

$$
C_{1} \leq \ldots \leq C_{n}
$$

holds.

Proof: Let $T_{k q}(t)$ be the total amount of time that machine $M_{q}$ spends on job $J_{k}$ in the time period $\left[0, t\left[\right.\right.$. Then, $f_{k}(t)=\sum_{q=1}^{m} T_{k q}(t) \cdot s_{q}$ is the part of job $J_{k}$ processed in $[0, t[$. Suppose that in some optimal schedule, there are two jobs $J_{i}$ and $J_{j}$ such that $r_{i}<r_{j}$ and $C_{i}>C_{j}$ holds. Since $0=f_{j}\left(r_{j}\right) \leq f_{i}\left(r_{j}\right)$ and $p=f_{j}\left(C_{j}\right)>f_{i}\left(C_{j}\right)$, there exists some time point $\xi \in\left[r_{j}, C_{j}\right]$ such that $f_{j}(\xi)=f_{i}(\xi)$, i.e. the part of job $J_{j}$ processed in $\left[\xi, C_{i}\right.$ [ is equal to the part of job $J_{i}$ processed in $\left[\xi, C_{i}\right.$ [. Now we can swap jobs $J_{i}$ and $J_{j}$ within the interval $\left[\xi, C_{i}[\right.$.

In fact, for the proposed algorithm this simple statement plays a crucial role. In the following, we only deal with schedules from the described class. Let $s^{*}$ be an optimal 
schedule for problem $Q\left|r_{j}, p_{j}=p, \operatorname{pmtn}\right| \sum C_{j}$ with $C_{1}\left(s^{*}\right) \leq \ldots \leq C_{n}\left(s^{*}\right)$. Each $C_{j}\left(s^{*}\right)$ belongs to some interval $\left[r_{i}, r_{i+1}\right]$. However, if we know for each $C_{j}\left(s^{*}\right)$ the corresponding interval $\left[r_{i}, r_{i+1}\right]$ such that $C_{j}\left(s^{*}\right) \in\left[r_{i}, r_{i+1}\right]$, then an optimal schedule can be easily found using a reduction to a network flow problem. Thus, the main question is to know the interval $\left[r_{i}, r_{i+1}\right]$ for each $C_{j}\left(s^{*}\right)$ such that $C_{j}\left(s^{*}\right) \in\left[r_{i}, r_{i+1}\right]$. However, this difficulty can be avoided due to criterion $\sum C_{j}$. For any job $J_{j}$, let the time interval $\left[r_{i}, r_{i+1}\right]$ be such that $C_{j} \in\left[r_{i}, r_{i+1}\right]$. Taking into account that $r_{1}=0$, we obtain

$$
C_{j}=\left(r_{2}-r_{1}\right)+\left(r_{3}-r_{2}\right)+\ldots+\left(r_{i}-r_{i-1}\right)+\left(C_{j}-r_{i}\right) .
$$

Due to this property, we introduce the completion time of job $J_{j}$ for each interval $\left[r_{i}, r_{i+1}\right]$. For each job $J_{j}$ with $j=1, \ldots, n$ and for each interval $\left[r_{i}, r_{i+1}\right]$ with $i=1, \ldots, n$, we define the value $C\left(J_{j}, r_{i}\right)$ such that $C\left(J_{j}, r_{i}\right)=C_{j}\left(s^{*}\right)$ if $C_{j}\left(s^{*}\right) \in\left[r_{i}, r_{i+1}\left[\right.\right.$, but if $C_{j}\left(s^{*}\right) \leq r_{i}$, then we set $C\left(J_{j}, r_{i}\right)=r_{i}$, and if $C_{j}\left(s^{*}\right) \geq r_{i+1}$, then we set $C\left(J_{j}, r_{i}\right)=r_{i+1}$. Thus, for job $J_{j}$ with $j=1, \ldots, n$, we have

$$
C\left(J_{j}, r_{i}\right)=\left\{\begin{array}{ccl}
C_{j}\left(s^{*}\right) & \text { if } & r_{i}<C_{j}\left(s^{*}\right)<r_{i+1} \\
r_{i} & \text { if } & C_{j}\left(s^{*}\right) \leq r_{i} \\
r_{i+1} & \text { if } & C_{j}\left(s^{*}\right) \geq r_{i+1}
\end{array}\right.
$$

So, for each $i=1, \ldots, n$, the values

$$
r_{i} \leq C\left(J_{1}, r_{i}\right) \leq \ldots \leq C\left(J_{i}, r_{i}\right) \leq C\left(J_{i+1}, r_{i}\right)=\ldots=C\left(J_{n}, r_{i}\right)=r_{i+1}
$$

define a partition of the interval $\left[r_{i}, r_{i+1}\right]$. Here, we set $r_{n+1}=r_{n}+n \cdot \max _{q}\left\{p / s_{q}\right\}$, i.e. $r_{n+1}$ is a time point after which no job will be processed.

In turn, each interval $\left[C\left(J_{j}, r_{i}\right), C\left(J_{j+1}, r_{i}\right)\right]$ is completely defined by the jobs processed in it. Thus, we denote by $v\left(J_{k}, M_{q}, J_{j}, r_{i}\right)$ the part of job $J_{k}$ processed in the interval $\left[C\left(J_{j-1}, r_{i}\right), C\left(J_{j}, r_{i}\right)\right]$ by machine $M_{q}$, i.e. the total processing time of job $J_{k}$ in the interval $\left[C\left(J_{j-1}, r_{i}\right), C\left(J_{j}, r_{i}\right)\right]$ equals $\frac{v\left(J_{k}, M_{q}, J_{j}, r_{i}\right)}{s_{k}}$ and for any job $J_{k}$, equality

$$
\sum_{q=1}^{m} \sum_{i=1}^{n} \sum_{j=1}^{n} v\left(J_{k}, M_{q}, J_{j}, r_{i}\right)=p
$$

holds.

The values $C\left(J_{j}, r_{i}\right)$, where $j=1, \ldots, n, \quad i=1, \ldots, n$, and the values $v\left(J_{k}, M_{q}, J_{j}, r_{i}\right)$, where $i, j=1, \ldots, n, \quad k=j, \ldots, i, \quad q=1, \ldots, m$, define a feasible solution of the following linear program. For convenience, we introduce $C\left(J_{0}, r_{i}\right)=r_{i}$. 


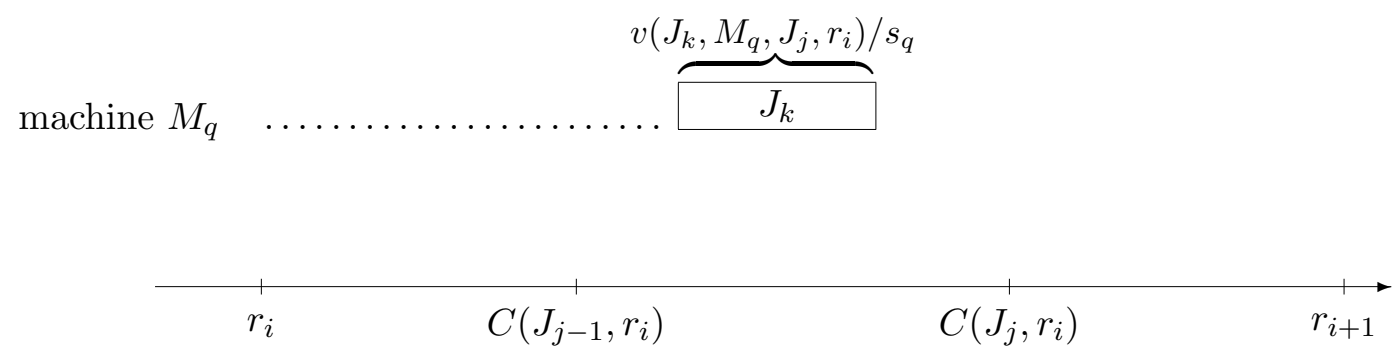

Figure 1: Here $v\left(J_{k}, M_{q}, J_{j}, r_{i}\right)$ is the part of job $J_{k}$ processed within $\left[C\left(J_{j-1}, r_{i}\right), C\left(J_{j}, r_{i}\right)\right]$.

Minimize

$$
\sum_{i=1}^{n}\left(\left(C\left(J_{1}, r_{i}\right)-r_{i}\right)+\ldots+\left(C\left(J_{n}, r_{i}\right)-r_{i}\right)\right)
$$

subject to

$$
\begin{aligned}
& r_{i}=C\left(J_{0}, r_{i}\right) \leq C\left(J_{1}, r_{i}\right) \leq \ldots \leq C\left(J_{i+1}, r_{i}\right)=\ldots \\
& =C\left(J_{n}, r_{i}\right)=r_{i+1}, \quad i=1, \ldots, n \\
& \sum_{q=1}^{m} \frac{v\left(J_{k}, M_{q}, J_{j}, r_{i}\right)}{s_{q}} \leq C\left(J_{j}, r_{i}\right)-C\left(J_{j-1}, r_{i}\right), \\
& i=1, \ldots, n, \quad j=1, \ldots, n, \quad k=1, \ldots, n \\
& \sum_{k=1}^{n} \frac{v\left(J_{k}, M_{q}, J_{j}, r_{i}\right)}{s_{q}} \leq C\left(J_{j}, r_{i}\right)-C\left(J_{j-1}, r_{i}\right) \\
& i=1, \ldots, n, \quad j=1, \ldots, n, \quad q=1, \ldots, m \\
& \sum_{i=1}^{n} \sum_{j=1}^{n} \sum_{q=1}^{m} v\left(J_{k}, M_{q}, J_{j}, r_{i}\right)=p, \quad k=1, \ldots, n \\
& v\left(J_{k}, M_{q}, J_{j}, r_{i}\right)=0 \quad i=1, \ldots, n-1, \quad j=i+1, \ldots, n, \\
& q=1, \ldots, m, \quad k=1, \ldots, n \\
& v\left(J_{k}, M_{q}, J_{j}, r_{i}\right)=0 \quad i=1, \ldots, n, \quad j=1, \ldots, i, \\
& q=1, \ldots, m, \quad k=1, \ldots, j-1 \\
& v\left(J_{k}, M_{q}, J_{j}, r_{i}\right)=0 \quad i=1, \ldots, n-1, \quad j=1, \ldots, i, \\
& q=1, \ldots, m, \quad k=i+1, \ldots, n
\end{aligned}
$$




$$
\begin{gathered}
C\left(J_{j}, r_{i}\right) \geq 0 \quad i=1, \ldots, n, \quad j=1, \ldots, n \\
v\left(J_{k}, M_{q}, J_{j}, r_{i}\right) \geq 0 \quad \begin{array}{l}
i=1, \ldots, n, \quad j=1, \ldots, n, \\
k=1, \ldots, n, \quad q=1, \ldots, m .
\end{array}
\end{gathered}
$$

The above formulation includes $O\left(m n^{3}\right)$ variables and constraints, i.e. this problem can be polynomially solved.

Note that:

- Constraints (2.3) hold since in $\left[r_{i}, r_{i+1}\right.$ [ only jobs $J_{1}, \ldots, J_{i}$ can be processed. Jobs $J_{i+1}, \ldots, J_{n}$ are available only after $r_{i+1}$.

- Constraints (2.4) hold since $\sum_{q=1}^{m} \frac{v\left(J_{k}, M_{q}, J_{j}, r_{i}\right)}{s_{q}}$ is the total time of processing job $J_{k}$ in the time interval $\left[C\left(J_{j-1}, r_{i}\right), C\left(J_{j}, r_{i}\right)[\right.$, see Figure 1.

- Inequalities (2.5) are true since $\sum_{k=1}^{n} \frac{v\left(J_{k}, M_{q}, J_{j}, r_{i}\right)}{s_{q}}$ is the total time when machine $M_{q}$ is busy in $\left[C\left(J_{j-1}, r_{i}\right), C\left(J_{j}, r_{i}\right)[\right.$. Note that constraints (2.4) and (2.5) provide the possibility to schedule all parts $v\left(J_{k}, M_{q}, J_{j}, r_{i}\right)$ within the time interval $\left[C\left(J_{j-1}, r_{i}\right), C\left(J_{j}, r_{i}\right)\right]$ in a feasible way.

- Constraints (2.6) hold since $\sum_{i=1}^{n} \sum_{j=1}^{n} \sum_{q=1}^{m} v\left(J_{k}, M_{q}, J_{j}, r_{i}\right)$ is the total part of job $J_{k}$ being processed.

- Constraints (2.7) hold since

$$
C\left(J_{i+1}, r_{i}\right)=C\left(J_{i+2}, r_{i}\right)=\ldots=C\left(J_{n}, r_{i}\right)=r_{i+1},
$$

i.e. there are no jobs processed in $\left[C\left(J_{i+1}, r_{i}\right), r_{i+1}[\right.$.

- Constraints (2.8) hold since jobs $J_{1}, \ldots, J_{j-1}$ cannot be processed after time point $C\left(J_{j-1}, r_{i}\right)$, see Figure 1.

- Constraints (2.9) hold since jobs $J_{i+1}, \ldots, J_{n}$ cannot be processed before time point $r_{i+1}$, see Figure 1.

- Furthermore, for schedule $s^{*}$ function (2.2) corresponds to the optimality criterion $\sum_{j=1}^{n} C_{j}$, since

$$
C\left(J_{j}, r_{i}\right)-r_{i}=\left\{\begin{array}{clc}
C_{j}\left(s^{*}\right)-r_{i} & \text { if } & r_{i}<C_{j}\left(s^{*}\right)<r_{i+1} \\
0 & \text { if } & C_{j}\left(s^{*}\right) \leq r_{i} \\
r_{i+1}-r_{i} & \text { if } & C_{j}\left(s^{*}\right) \geq r_{i+1}
\end{array}\right.
$$

and therefore, $\sum_{i=1}^{n}\left(C\left(J_{j}, r_{i}\right)-r_{i}\right)=C_{j}\left(s^{*}\right)$.

Thus, $\sum_{i=1}^{n}\left(\left(C\left(J_{1}, r_{i}\right)-r_{i}\right)+\ldots+\left(C\left(J_{n}, r_{i}\right)-r_{i}\right)\right)=\sum_{j=1}^{n} C_{j}\left(s^{*}\right)$. 
Summarizing, we have proven

Theorem 1 For any optimal schedule $s^{*}$ of problem $Q \mid r_{j}, p_{j}=p$, pmtn $\mid \sum C_{j}$, there is a corresponding feasible solution of (2.2)-(2.11) such that $C_{1}\left(s^{*}\right) \leq \ldots \leq C_{n}\left(s^{*}\right)$ and

$$
\sum_{j=1}^{n} C_{j}\left(s^{*}\right)=\sum_{i=1}^{n}\left(\left(C\left(J_{1}, r_{i}\right)-r_{i}\right)+\ldots+\left(C\left(J_{n}, r_{i}\right)-r_{i}\right)\right)
$$

hold.

The next theorem shows that conversely any feasible solution of (2.2)-(2.11) also provides a feasible schedule.

Theorem 2 Any feasible solution of problem (2.2)-(2.11) provides a feasible schedule $s^{*}$ for the scheduling problem $Q \mid r_{j}, p_{j}=p$, pmtn $\mid \sum C_{j}$ such that

$$
\sum_{j=1}^{n} C_{j}\left(s^{*}\right)=\sum_{i=1}^{n}\left(\left(C\left(J_{1}, r_{i}\right)-r_{i}\right)+\ldots+\left(C\left(J_{n}, r_{i}\right)-r_{i}\right)\right)
$$

holds.

Proof: Any feasible solution of (2.2)-(2.11) provides some values $C\left(J_{j}, r_{i}\right)$ and $v\left(J_{k}\right.$, $\left.M_{q}, J_{j}, r_{i}\right)$, and hence it provides some feasible schedule, which can be reconstructed by processing all parts $v\left(J_{k}, M_{q}, J_{j}, r_{i}\right)$ in the intervals $\left[C\left(J_{j-1}, r_{i}\right), C\left(J_{j}, r_{i}\right)[\right.$. If the values $C\left(J_{j}, r_{i}\right)$ obtained are such that for any job $J_{j}$, there is an index $e$ such that $C\left(J_{j}, r_{i}\right)=r_{i+1}$ holds for any $r_{i}<r_{e}$ and $C\left(J_{j}, r_{i}\right)=r_{i}$ holds for any $r_{i}>r_{e}$, then for the schedule $s^{*}$ defined by $C\left(J_{j}, r_{i}\right)$ and $v\left(J_{k}, M_{q}, J_{j}, r_{i}\right)$ equality

$$
\sum_{j=1}^{n} C_{j}\left(s^{*}\right)=\sum_{i=1}^{n}\left(\left(C\left(J_{1}, r_{i}\right)-r_{i}\right)+\ldots+\left(C\left(J_{n}, r_{i}\right)-r_{i}\right)\right)
$$

holds. Thus, in this case Theorem 2 is true.

Now suppose that for some job $J_{j}$, there does not exist such an index $e$. In this case, one can find two intervals $\left[r_{k}, r_{k+1}\right.$ [ and $\left[r_{h}, r_{h+1}\right.$ [ such that $r_{k} \leq C\left(J_{j}, r_{k}\right)<r_{k+1} \leq r_{h}<$ $C\left(J_{j}, r_{h}\right) \leq r_{h+1}$ holds. Transform the schedule $s^{*}$ in the following way. Take the largest value of $\delta$ such that in $\left[C\left(J_{j}, r_{k}\right), C\left(J_{j}, r_{k}\right)+\delta\right]$ and in $\left[C\left(J_{j}, r_{h}\right), C\left(J_{j}, r_{h}\right)-\delta\right]$, each machine is either idle or processes exactly one job. Now we swap $J_{j}$ from the interval $\left[C\left(J_{j}, r_{h}\right), C\left(J_{j}, r_{h}\right)-\delta\right]$ and $J_{l}$ (if any) from the interval $\left[C\left(J_{j}, r_{k}\right), C\left(J_{j}, r_{k}\right)+\delta\right]$ on the same machine, say $M_{z}$ (see Figure 2). Since in $\left[r_{k}, r_{k+1}\right.$ [ inequality $C\left(J_{l}, r_{k}\right)>C\left(J_{j}, r_{k}\right)$ holds, it follows that inequality $r_{j} \leq r_{l}$ holds. This implies $C\left(J_{l}, r_{h}\right) \geq C\left(J_{j}, r_{h}\right)$ and $\sum C_{j}$ does not change.

Now, if it happens that $J_{l}$ is processed in $\left[C\left(J_{j}, r_{h}\right), C\left(J_{j}, r_{h}\right)-\delta\right]$ by some other machine, say $M_{g} \neq M_{z}$, then we swap job $J_{l}$ from $\left[C\left(J_{j}, r_{h}\right), C\left(J_{j}, r_{h}\right)-\delta\right]$ and $J_{f}$ (if any) from 


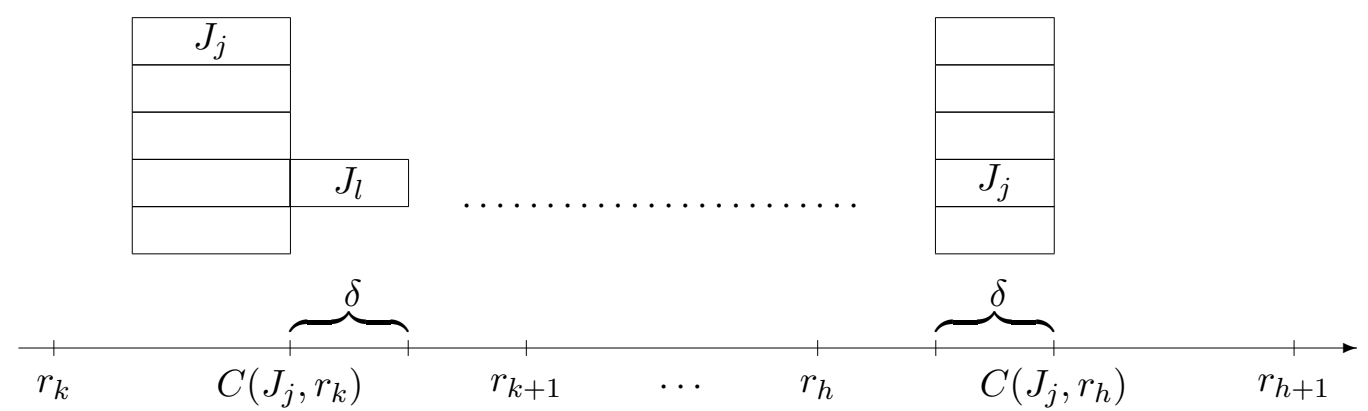

Figure 2: Swap of $J_{j}$ from $\left[C\left(J_{j}, r_{h}\right), C\left(J_{j}, r_{h}\right)-\delta\right]$ and $J_{l}$ from $\left[C\left(J_{j}, r_{k}\right), C\left(J_{j}, r_{k}\right)+\delta\right]$.

$\left[C\left(J_{j}, r_{k}\right), C\left(J_{j}, r_{k}\right)+\delta\right]$ on machine $M_{g}$. We will continue with this swapping as long as the schedule remains infeasible. Note that the value of (2.2) does not increase.

Example. Let us consider the instance from [2]. The data are the following: $m=3$, $p=10, r_{1}=\ldots=r_{5}=0, r_{6}=r_{7}=11, r_{8}=r_{9}=18, r_{10}=22, r_{11}=27$. Let the speeds be $s_{1}=1, s_{2}=1$, and $s_{3}=2$. Using CPLEX ${ }^{2}$, we solve the corresponding LP problem. We obtain an optimal solution with the objective function value 196.54296875 and with the following non-zero variables:

\begin{tabular}{|c|c|c|c|}
\hline $\begin{array}{l}C\left(J_{0}, r_{6}\right)=11 \\
C\left(J_{0}, r_{10}\right)=22 \\
C\left(J_{0}, r_{8}\right)=18 \\
C\left(J_{1}, r_{5}\right)=5 \\
C\left(J_{1}, r_{9}\right)=18 \\
C\left(J_{2}, r_{6}\right)=11 \\
C\left(J_{2}, r_{10}\right)=22 \\
C\left(J_{3}, r_{7}\right)=11 \\
C\left(J_{3}, r_{11}\right)=27 \\
C\left(J_{4}, r_{8}\right)=18 \\
C\left(J_{5}, r_{5}\right)=11 \\
C\left(J_{5}, r_{9}\right)=18 \\
C\left(J_{6}, r_{6}\right)=11 \\
C\left(J_{6}, r_{10}\right)=22 \\
C\left(J_{7}, r_{7}\right)=18 \\
C\left(J_{7}, r_{11}\right)=27 \\
C\left(J_{8}, r_{8}\right)=18 \\
C\left(J_{9}, r_{5}\right)=11 \\
C\left(J_{9}, r_{9}\right)=22 \\
C\left(J_{10}, r_{6}\right)=11 \\
C\left(J_{10}, r_{10}\right)=27 \\
C\left(J_{11}, r_{7}\right)=18\end{array}$ & $\begin{array}{l}C\left(J_{0}, r_{7}\right)=11 \\
C\left(J_{0}, r_{11}\right)=27 \\
C\left(J_{0}, r_{9}\right)=18 \\
C\left(J_{1}, r_{6}\right)=11 \\
C\left(J_{1}, r_{10}\right)=22 \\
C\left(J_{2}, r_{7}\right)=11 \\
C\left(J_{2}, r_{11}\right)=27 \\
C\left(J_{3}, r_{8}\right)=18 \\
C\left(J_{4}, r_{5}\right)=11 \\
C\left(J_{4}, r_{9}\right)=18 \\
C\left(J_{5}, r_{6}\right)=11 \\
C\left(J_{5}, r_{10}\right)=22 \\
C\left(J_{6}, r_{7}\right)=17.625 \\
C\left(J_{6}, r_{11}\right)=27 \\
C\left(J_{7}, r_{8}\right)=18 \\
C\left(J_{8}, r_{5}\right)=11 \\
C\left(J_{8}, r_{9}\right)=22 \\
C\left(J_{9}, r_{6}\right)=11 \\
C\left(J_{9}, r_{10}\right)=23.9140625 \\
C\left(J_{10}, r_{7}\right)=18 \\
C\left(J_{10}, r_{11}\right)=28.9140625 \\
C\left(J_{11}, r_{8}\right)=18\end{array}$ & $\begin{array}{l}C\left(J_{0}, r_{8}\right)=18 \\
C\left(J_{0}, r_{6}\right)=11 \\
C\left(J_{0}, r_{10}\right)=22 \\
C\left(J_{1}, r_{7}\right)=11 \\
C\left(J_{1}, r_{11}\right)=27 \\
C\left(J_{2}, r_{8}\right)=18 \\
C\left(J_{3}, r_{5}\right)=10.5 \\
C\left(J_{3}, r_{9}\right)=18 \\
C\left(J_{4}, r_{6}\right)=11 \\
C\left(J_{4}, r_{10}\right)=22 \\
C\left(J_{5}, r_{7}\right)=14.25 \\
C\left(J_{5}, r_{11}\right)=27 \\
C\left(J_{6}, r_{8}\right)=18 \\
C\left(J_{7}, r_{5}\right)=11 \\
C\left(J_{7}, r_{9}\right)=19.3125 \\
C\left(J_{8}, r_{6}\right)=11 \\
C\left(J_{8}, r_{10}\right)=23.65625 \\
C\left(J_{9}, r_{7}\right)=18 \\
C\left(J_{9}, r_{11}\right)=28.9140625 \\
C\left(J_{10}, r_{8}\right)=18 \\
C\left(J_{11}, r_{5}\right)=11 \\
C\left(J_{11}, r_{9}\right)=22\end{array}$ & $\begin{array}{l}C\left(J_{0}, r_{9}\right)=18, \\
C\left(J_{0}, r_{7}\right)=11, \\
C\left(J_{0}, r_{11}\right)=27, \\
C\left(J_{1}, r_{8}\right)=18, \\
C\left(J_{2}, r_{5}\right)=7.5, \\
C\left(J_{2}, r_{9}\right)=18, \\
C\left(J_{3}, r_{6}\right)=11, \\
C\left(J_{3}, r_{10}\right)=22, \\
C\left(J_{4}, r_{7}\right)=11, \\
C\left(J_{4}, r_{11}\right)=27, \\
C\left(J_{5}, r_{8}\right)=18, \\
C\left(J_{6}, r_{5}\right)=11, \\
C\left(J_{6}, r_{9}\right)=18, \\
C\left(J_{7}, r_{6}\right)=11, \\
C\left(J_{7}, r_{10}\right)=22, \\
C\left(J_{8}, r_{7}\right)=18, \\
C\left(J_{8}, r_{11}\right)=27, \\
C\left(J_{9}, r_{8}\right)=18, \\
C\left(J_{10}, r_{5}\right)=11, \\
C\left(J_{10}, r_{9}\right)=22, \\
C\left(J_{11}, r_{6}\right)=11, \\
C\left(J_{11}, r_{10}\right)=27,\end{array}$ \\
\hline
\end{tabular}

\footnotetext{
${ }^{2}$ CPLEX is a trademark of ILOG, Inc. http://www.ilog.com/products/cplex/
} 


$v\left(J_{1}, M_{3}, J_{1}, r_{5}\right)=10$
$v\left(J_{3}, M_{1}, J_{1}, r_{5}\right)=1.5$
$v\left(J_{3}, M_{1}, J_{2}, r_{5}\right)=2.5$
$v\left(J_{4}, M_{1}, J_{3}, r_{5}\right)=3$
$v\left(J_{5}, M_{1}, J_{4}, r_{5}\right)=0.5$
$v\left(J_{7}, M_{1}, J_{5}, r_{7}\right)=3.25$
$v\left(J_{7}, M_{3}, J_{7}, r_{7}\right)=0.75$
$v\left(J_{9}, M_{1}, J_{7}, r_{9}\right)=1.3125$
$v\left(J_{8}, M_{3}, J_{8}, r_{10}\right)=3.3125$
$v\left(J_{9}, M_{3}, J_{9}, r_{10}\right)=0.515625$
$v\left(J_{9}, M_{3}, J_{9}, r_{11}\right)=3.828125$,
$v\left(J_{11}, M_{3}, J_{11}, r_{11}\right)=8.0859375$.

$v\left(J_{1}, M_{3}, J_{1}, r_{5}\right)=10$,

$v\left(J_{3}, M_{1}, J_{2}, r_{5}\right)=2.5$

$v\left(J_{5}, M_{1}, J_{4}, r_{5}\right)=0.5$

$v\left(J_{7}, M_{1}, J_{5}, r_{7}\right)=3.25$,

$v\left(J_{7}, M_{3}, J_{7}, r_{7}\right)=0.75$,

$v\left(J_{9}, M_{1}, J_{7}, r_{9}\right)=1.3125$,

$v\left(J_{8}, M_{3}, J_{8}, r_{10}\right)=3.3125$

$v\left(J_{9}, M_{3}, J_{9}, r_{11}\right)=3.828125$

$v\left(J_{11}, M_{3}, J_{11}, r_{11}\right)=8.0859375$.
$v\left(J_{2}, M_{1}, J_{1}, r_{5}\right)=3.5$,

$v\left(J_{4}, M_{2}, J_{1}, r_{5}\right)=3.5$,

$v\left(J_{4}, M_{2}, J_{2}, r_{5}\right)=2.5$,

$v\left(J_{5}, M_{2}, J_{3}, r_{5}\right)=3$,

$v\left(J_{5}, M_{3}, J_{5}, r_{7}\right)=6.5$,

$v\left(J_{6}, M_{3}, J_{6}, r_{7}\right)=6.75$,

$v\left(J_{7}, M_{3}, J_{7}, r_{9}\right)=2.625$,

$v\left(J_{8}, M_{3}, J_{8}, r_{9}\right)=5.375$,

$v\left(J_{9}, M_{1}, J_{8}, r_{10}\right)=1.65625$,

$v\left(J_{10}, M_{1}, J_{9}, r_{10}\right)=0.2578125$,

$v\left(J_{10}, M_{1}, J_{9}, r_{11}\right)=1.9140625$,
$v\left(J_{2}, M_{2}, J_{1}, r_{5}\right)=1.5$,

$v\left(J_{2}, M_{3}, J_{2}, r_{5}\right)=5$,

$v\left(J_{3}, M_{3}, J_{3}, r_{5}\right)=6$,

$v\left(J_{4}, M_{3}, J_{4}, r_{5}\right)=1$,

$v\left(J_{6}, M_{2}, J_{5}, r_{7}\right)=3.25$,

$v\left(J_{7}, M_{1}, J_{6}, r_{7}\right)=3.375$,

$v\left(J_{8}, M_{2}, J_{7}, r_{9}\right)=1.3125$,

$v\left(J_{9}, M_{2}, J_{8}, r_{9}\right)=2.6875$,

$v\left(J_{10}, M_{2}, J_{8}, r_{10}\right)=1.65625$,

$v\left(J_{10}, M_{3}, J_{10}, r_{10}\right)=6.171875$,

$v\left(J_{11}, M_{2}, J_{9}, r_{11}\right)=1.9140625$,

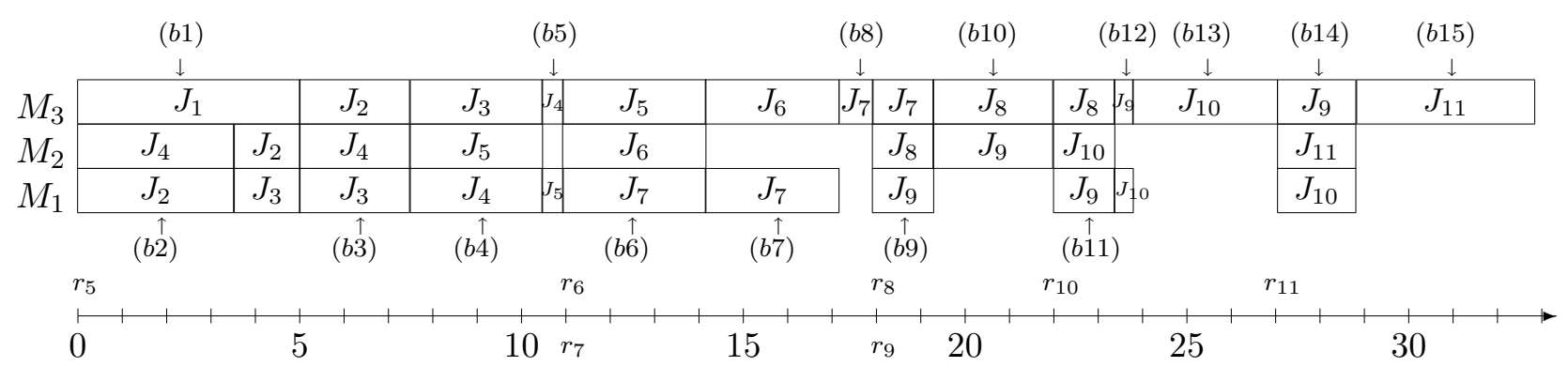

Figure 3: The optimal schedule corresponding to the CPLEX solution. Here (b1) corresponds to $v\left(J_{1}, M_{3}, J_{1}, r_{5}\right)=10,(b 2)$ corresponds to $v\left(J_{2}, M_{1}, J_{1}, r_{5}\right)=3.5$, (b3) corresponds to $v\left(J_{3}, M_{1}, J_{2}, r_{5}\right)=$ 2.5 , (b4) corresponds to $v\left(J_{4}, M_{1}, J_{3}, r_{5}\right)=3$, (b5) corresponds to $v\left(J_{4}, M_{3}, J_{4}, r_{5}\right)=1$, (b6) corresponds to $v\left(J_{7}, M_{1}, J_{5}, r_{7}\right)=3.25$, (b7) corresponds to $v\left(J_{7}, M_{1}, J_{6}, r_{7}\right)=3.375$, (b8) corresponds to $v\left(J_{7}, M_{3}, J_{7}, r_{7}\right)=0.75$, (b9) corresponds to $v\left(J_{9}, M_{1}, J_{7}, r_{9}\right)=1.3125,(b 10)$ corresponds to $v\left(J_{8}, M_{3}, J_{8}, r_{9}\right)=5.375$, (b11) corresponds to $v\left(J_{9}, M_{1}, J_{8}, r_{10}\right)=1.65625$, (b12) corresponds to $v\left(J_{9}, M_{3}, J_{9}, r_{10}\right)=0.515625$, (b13) corresponds to $v\left(J_{10}, M_{3}, J_{10}, r_{10}\right)=6.171875$, (b14) corresponds to $v\left(J_{9}, M_{3}, J_{9}, r_{11}\right)=3.828125,(b 15)$ corresponds to $v\left(J_{11}, M_{3}, J_{11}, r_{11}\right)=8.0859375$.

The solution is defined by the five non-zero intervals $\left[r_{5}, r_{6}\left[=\left[0,11\left[,\left[r_{7}, r_{8}[=[11,18[\right.\right.\right.\right.\right.$, $\left[r_{9}, r_{10}\left[=\left[18,22\left[,\left[r_{10}, r_{11}\left[=\left[22,27\left[\right.\right.\right.\right.\right.\right.\right.\right.$, and $\left[r_{11}, r_{12}\left[=\left[27,137\left[\right.\right.\right.\right.$. For $\left[r_{5}, r_{6}\left[\right.\right.$, we get $C\left(J_{1}, r_{5}\right)=$ $5, C\left(J_{2}, r_{5}\right)=7.5, C\left(J_{3}, r_{5}\right)=10.5, C\left(J_{4}, r_{5}\right)=11$. For $\left[r_{7}, r_{8}\left[=\left[11,18\left[\right.\right.\right.\right.$, we get $C\left(J_{4}, r_{7}\right)=$ $11, C\left(J_{5}, r_{7}\right)=14.25, C\left(J_{6}, r_{7}\right)=17.625, C\left(J_{7}, r_{7}\right)=18$. For $\left[r_{9}, r_{10}[=[18,22[\right.$, we have $C\left(J_{6}, r_{9}\right)=18, C\left(J_{7}, r_{9}\right)=19.3125, C\left(J_{8}, r_{9}\right)=22, C\left(J_{9}, r_{9}\right)=22$. For $\left[r_{10}, r_{11}[=[22,27[\right.$, we have $C\left(J_{7}, r_{10}\right)=22, C\left(J_{8}, r_{10}\right)=23.65625, C\left(J_{9}, r_{10}\right)=23.9140625, C\left(J_{10}, r_{10}\right)=27$. For $\left[r_{11}, r_{12}\left[=\left[27,137\left[\right.\right.\right.\right.$, we have $C\left(J_{8}, r_{11}\right)=27, C\left(J_{9}, r_{11}\right)=28.9140625, C\left(J_{10}, r_{11}\right)=$ $28.9140625, C\left(J_{11}, r_{11}\right)=32.95703125$.

In Figure 4, we leave only blocks corresponding to such values $v\left(J_{k}, M_{q}, J_{j}, r_{i}\right)$ that define the $C_{j}$ values for each interval $\left[r_{i}, r_{i+1}[\right.$.

From Figure 4, one can see that (2.1) holds for each job except job $J_{9}$, since $r_{10}<$ $C\left(J_{9}, r_{10}\right)<r_{11}$ and $r_{11}<C\left(J_{9}, r_{11}\right)<r_{12}$. Therefore, for all jobs except job $J_{9}$, we have $C_{1}=C\left(J_{1}, r_{5}\right)=5, C_{2}=C\left(J_{2}, r_{5}\right)=7.5, C_{3}=C\left(J_{3}, r_{5}\right)=10.5, C_{4}=C\left(J_{4}, r_{5}\right)=11$, $C_{5}=C\left(J_{5}, r_{7}\right)=14.25, C_{6}=C\left(J_{6}, r_{7}\right)=17.625, C_{7}=C\left(J_{7}, r_{9}\right)=19.3125, C_{8}=$ 


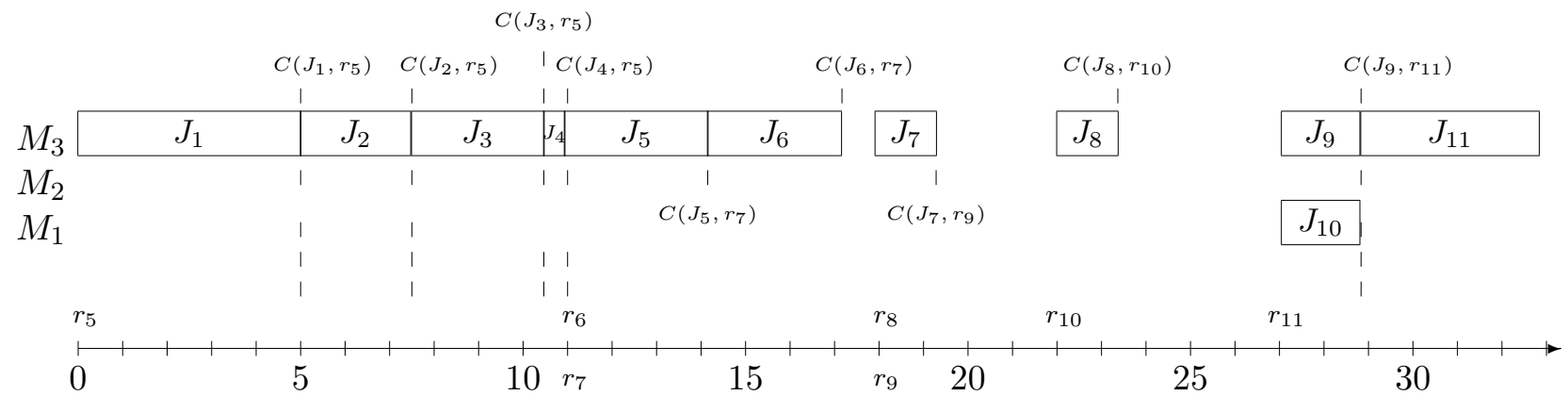

Figure 4: Here each block corresponds to such a value $v\left(J_{k}, M_{q}, J_{j}, r_{i}\right)$ that defines $C\left(J_{j}, r_{i}\right)$.

$C\left(J_{8}, r_{10}\right)=23.65625, C_{10}=C\left(J_{10}, r_{11}\right)=28.9140625, C_{11}=C\left(J_{11}, r_{11}\right)=32.95703125$. For job $J_{9}$, see Figure 3 , we have $C_{9}=C\left(J_{9}, r_{11}\right)=28.9140625$, but from (2.2) we calculate the value

$$
\begin{aligned}
C_{9}= & \sum_{i=1}^{11}\left(C\left(J_{9}, r_{i}\right)-r_{i}\right) \\
= & \left(C\left(J_{9}, r_{1}\right)-r_{1}\right)+\left(C\left(J_{9}, r_{2}\right)-r_{2}\right)+\left(C\left(J_{9}, r_{3}\right)-r_{3}\right)+\left(C\left(J_{9}, r_{4}\right)-r_{4}\right)+ \\
& \left(C\left(J_{9}, r_{5}\right)-r_{5}\right)+\left(C\left(J_{9}, r_{6}\right)-r_{6}\right)+\left(C\left(J_{9}, r_{7}\right)-r_{7}\right)+\left(C\left(J_{9}, r_{8}\right)-r_{8}\right)+ \\
& \left(C\left(J_{9}, r_{9}\right)-r_{9}\right)+\left(C\left(J_{9}, r_{10}\right)-r_{10}\right)+\left(C\left(J_{9}, r_{11}\right)-r_{11}\right) \\
= & (0-0)+(0-0)+(0-0)+(0-0)+(11-0)+(11-11)+(18-11)+ \\
& (18-18)+(22-18)+(23.9140625-22)+(28.9140625-27) \\
= & 25.828125 .
\end{aligned}
$$

To get the schedule with $C_{9}=25.828125$, one can apply the transformation described in the proof of Theorem 2. In the first step, we swap a part of job $J_{9}$ with length $\delta$ and a part of job $J_{10}$ with length $\delta$ on machine $M_{3}$, see Figure 5 . After the first step, we

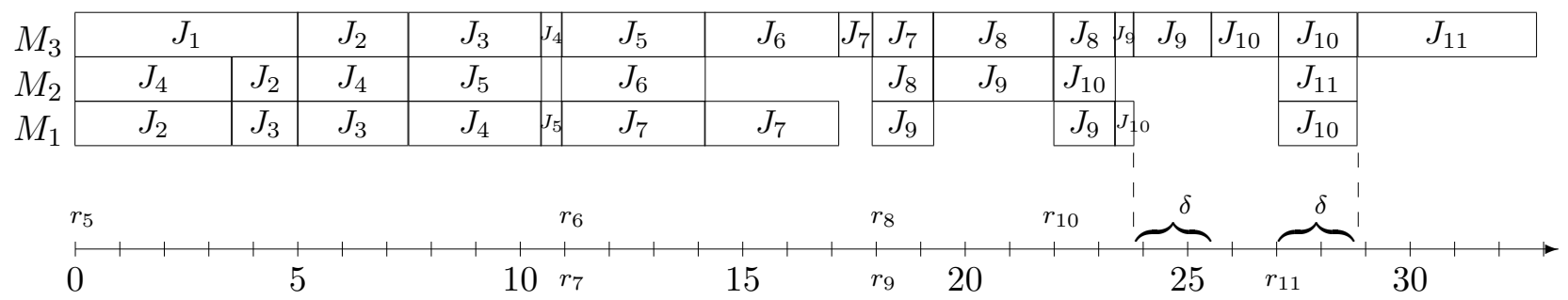

Figure 5: The schedule obtained after the first step of the transformation.

obtain an infeasible schedule, see Figure 5 , since job $J_{10}$ is processed on machine $M_{3}$ and machine $M_{1}$ at the same time. Nevertheless, the value of (2.2) is not changed. In the 
next step of the transformation, we swap a part of job $J_{10}$ with length $\delta$ with the idle interval of length $\delta$ on machine $M_{1}$, see Figure 6 . The obtained schedule is feasible and the value of $(2.2)$ is not changed, but now we have

$$
C_{9}=C\left(J_{9}, r_{10}\right)+\frac{1}{2} \cdot v\left(J_{9}, M_{3}, J_{9}, r_{11}\right)=23.9140625+\frac{1}{2} \cdot 3.828125=25.828125
$$

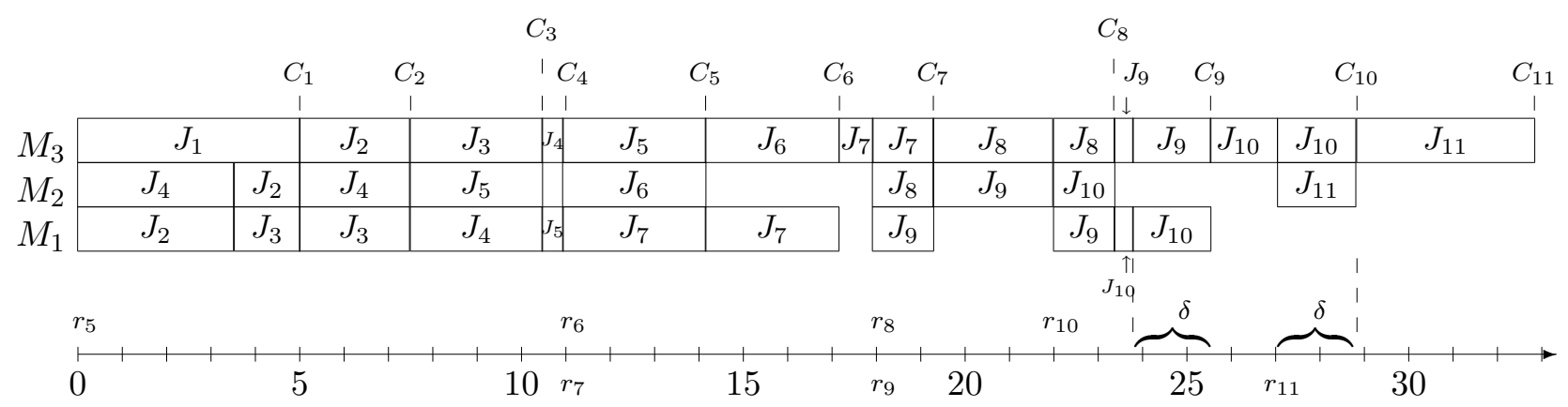

Figure 6: The schedule obtained after the second step of the transformation.

Thus, one can see that we do not need to apply the transformation. Since as a result of solving (2.2)-(2.11) we obtain the values $C_{1}, \ldots, C_{n}$, we can reconstruct the optimal schedule by solving the following network flow problem, see [4].

Suppose that all points $r_{1}, \ldots, r_{n}, C_{1}, \ldots, C_{n}$ are enumerated in non-decreasing order of their values, say $t_{1} \leq \ldots \leq t_{k}$. We construct a network which has the following vertices:

- a source $u$ and a sink $w$,

- job-vertices $\left\{J_{j} \mid j=1, \ldots, n\right\}$,

- machine-time-interval-vertices $\left\{\left(t_{i}, t_{i+1}, M_{e}\right) \mid i=1, \ldots, k-1, \quad e=1, \ldots, m\right\}$

The arcs in the network defined are the following:

- for each job-vertex $J_{j}$, there is an $\operatorname{arc}\left(u, J_{j}\right)$ with the capacity $p$, and there is an $\operatorname{arc}\left(J_{j},\left(t_{i}, t_{i+1}, M_{e}\right)\right)$ if $r_{j} \leq t_{i}$ and $t_{i+1} \leq C_{j}$ hold,

- for each vertex $\left(t_{i}, t_{i+1}, M_{e}\right)$, there is an $\operatorname{arc}\left(\left(t_{i}, t_{i+1}, M_{e}\right), w\right)$ with the capacity $\left(t_{i+1}-t_{i}\right) \cdot s_{e}$.

Any maximal flow will reconstruct an optimal schedule.

Thus, to solve problem $Q \mid r_{j}, p_{j}=p$, pmtn $\mid \sum C_{j}$, one has to do the following: 
1. Solve the corresponding linear program (2.2)-(2.11), and calculate the values $C_{j}=\sum_{i=1}^{n}\left(C\left(J_{j}, r_{i}\right)-r_{i}\right)$ for each job $J_{j}$.

2. Reconstruct an optimal schedule by solving the corresponding network flow problem.

\section{Concluding remarks}

In this paper, we considered problem $Q \mid r_{j}, p_{j}=p$, pmtn $\mid \sum C_{j}$, whose complexity status was open yet. We presented a polynomial algorithm which is based on the solution of a linear program and a network flow problem. For further research, the most interesting question is whether it is possible to simplify the proposed linear programming formulation in the same way as it was made in [1] for problem $P\left|r_{j}, p_{j}=p, \operatorname{pmtn}\right| \sum C_{j}$. In particular, is it possible to find such special properties of an optimal schedule which permit to reduce the size of the linear programming formulation?

\section{References}

[1] Ph. Baptiste, P. Brucker, M. Chrobak, C. Dürr, S.A. Kravchenko, F. Sourd, The complexity of mean flow time scheduling problems with release times, Journal of Scheduling 10 (2007) 139-146.

[2] P. Brucker, S.A. Kravchenko, Polynomial algorithm for parallel machine mean flow time scheduling problem with release dates, Computational Science and Its Applications (ICCSA 2005) (O.Gervasi, M.L. Gavrilova eds.), 2005, Lecture Notes in Computer Science 3483, Springer: Berlin, 182-191.

[3] J. Labetoulle, E.L. Lawler, J.K. Lenstra, A.H.G. Rinnooy Kan, Preemptive scheduling of uniform machines subject to release dates, Progress in Combinatorial Optimization, (H.R. Pulleyblank ed.), 1984, Academic Press: New York, 245-261.

[4] E.L. Lawler, Combinatorial Optimization: Networks and Matroids. New York: Holt, Rinehart and Winston, 1976.

[5] E.L. Lawler, J. Labetoulle, On preemptive scheduling on unrelated parallel processors by linear programming, J. Assoc. Comput. Mach. 25 (1978) 612-619.

[6] R. Sitters, Complexity of preemptive minsum scheduling on unrelated parallel machines, Journal of Algorithms 57 (2005) 37-48. 